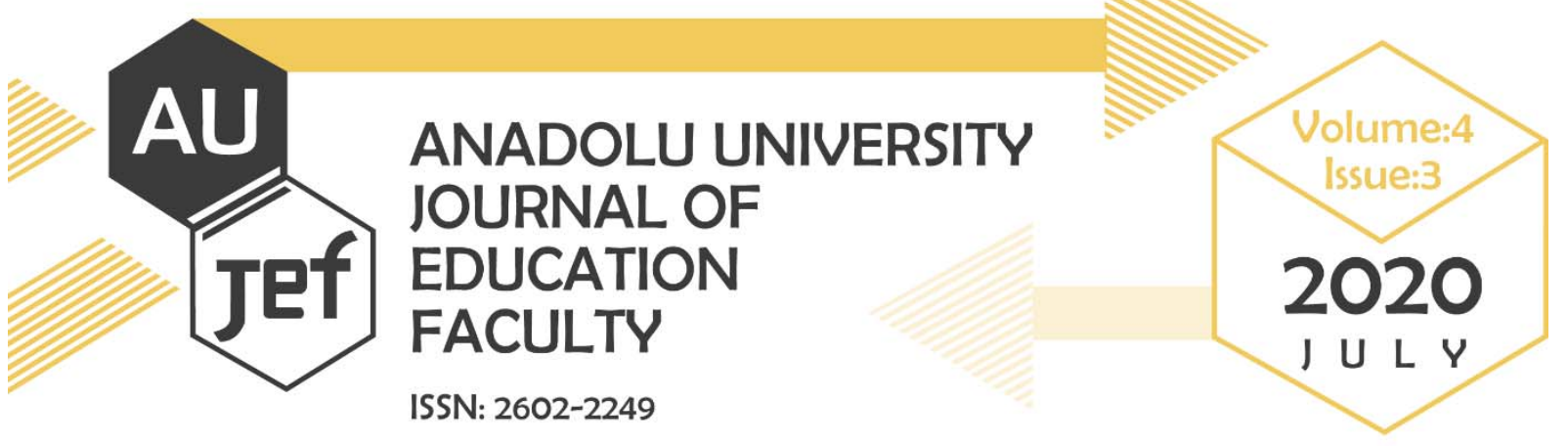

La gestion des comportements perturbateurs des élèves du secondaire par les enseignants stagiaires tunisiens en éducation physique : effets d'un programme de formation en prévention et en gestion de l'indiscipline

\title{
Prevention and management of high school pupils' undisciplined behaviors: Effects of a complementary training program intented for Tunisian physical education teacher trainees'
}

Talel MADDEH ${ }^{1}$

Salem AMAMOU ${ }^{2}$

Jean-François DESBIENS ${ }^{3}$

Nizar SOUISSI ${ }^{4}$

Type d'article: Article de recherche

Date de la demande: 14.01 .2020

Date d'acceptation: 30.06 .2020

Citer: Madeh, T., Amamou, S., Desbiens, J-F. \& Souissi, N. (2020). La gestion des comportements perturbateurs des élèves du secondaire par les enseignants stagiaires tunisiens en éducation physique : effets d'un programme de formation en prévention et en gestion de l'indiscipline. Anadolu University Journal of Education Faculty (AUJEF), 4(3), 323-344.

RÉSUMÉ: En Tunisie, la formation préparatoire à l'enseignement de l'éducation physique (EP) ne compte aucun cours spécifiquement dédié à la prévention ainsi qu'à la gestion des comportements d'indiscipline (CI). Devant ce constat, il est apparu pertinent d'élaborer et de mettre en œuvre une formation complémentaire en prévention et en gestion de l'indiscipline s'appuyant sur le modèle de Sieber (2001). Cet article a pour objectif général de décrire et d'analyser les effets d'une formation complémentaire sur la compétence d'enseignants stagiaires tunisiens en EP à prévenir et à gérer l'indiscipline. Pour ce faire, recherche quantitative adoptant un devis quasi-expérimental en trois temps basé sur des analyses vidéoscopiques en différé a été réalisée sur deux groupes de stagiaires : un groupe expérimental $\left(\mathrm{Gr}_{\mathrm{Exp}} ; \mathrm{n}=5\right)$ et un groupe témoin $\left(\mathrm{Gr}_{\mathrm{Tém}} ; \mathrm{n}=5\right)$. Les résultats obtenus indiquent une diminution de l'apparition des CI et des fractures de l'enseignement dans les cours des stagiaires du $\mathrm{Gr}_{\text {Exp. }}$. Ce constat pourrait être expliqué par une nette amélioration du $\mathrm{Gr}_{\text {Exp }}$ comparativement au $\mathrm{Gr}_{\text {Tém }}$ quant à sa compétence de prévenir et gérer l'indiscipline. Dans cette perspective, l'étude encourage les institutions de formation à l'enseignement à bonifier et à réviser leur curriculum de formation afin de mieux préparer les enseignants à composer de façon stratégique avec les CI des élèves.

Mots clés: Formation complémentaire, enseignants stagiaires, prévention et gestion de l'indiscipline, éducation physique.

\footnotetext{
${ }^{1}$ Auteur correspondant, talelmaddeh@hotmail.fr, ORCID: 0000-0001-6476-9351

${ }^{2}$ ORCID: 0000-0001-6847-0250

${ }^{3}$ ORCID: 0000-0003-4668-8675

${ }^{4}$ ORCID: 0000-0003-1227-8221
} 
ABSTRACT: In Tunisia, the preparatory training for the teaching of physical education (PE) does not include any course which is specifically dedicated to the prevention as well as the management of undisciplined behaviors (UB) (Maddeh et al, 2015). Accordingly, it was considered appropriate to implement an additional training to prevent and manage indiscipline based on the Sieber model (2001). The aim of this article is to describe and analyze the effects of complimentary training on the competence of Tunisian trainees teachers in PE to avoid and manage indiscipline. For this purpose, a quantitative quasi-experiental study based ont repeated videoscopic analysis was carried out on two groups of trainees: experimental groups' (exp.GR) $(n=5)$ and a control groups' (control.GR) $(\mathrm{n}=5)$. The obtained results show a decrease in the appearance of UB and teaching fractures in trainees' course (exp. Gr). This could be explained by an important improvement in exp.Gr compared to the controlGr in terms of ability to prevent and manage indiscipline. Accordingly, the study encourages teacher training institutions to boost and revise their training curriculum in order to better strategically prepare teachers to compose with the pupils' UB.

Keywords: Complimentary training, trainee teachers, prevention and management of indiscipline, physical education. 


\section{INTRODUCTION}

Les recherches contemporaines en enseignement (Archambault \& Chouinard, 2016; Gauthier, Desbiens, Malo, Martineau et Simard, 1997; Nault \& Lacourse, 2016; Levin \& Nolan, 2000) montrent depuis une bonne quarantaine d'années que la gestion de classe est une fonction déterminante en ce qui a trait à l'efficacité de l'enseignement. D'ailleurs, de nombreux travaux (Gaudreau, 2017; Hattie, 2012; Ministère de l'Éducation, de l'enseignement supérieur et de la recherche (MEESR), 2015) rapportent une relation significative entre la gestion de classe efficace et la qualité de l'environnement d'apprentissage offert aux élèves. Pour Evertson \& Weinstein (2006, p. 4; traduction libre) : « La gestion de classe désigne l'ensemble des démarches ou des actions entreprises par les enseignants en vue de créer un environnement propice aux apprentissages ». De leur côté, Nault \& Fijalkow (1999, p. 453) avancent que la gestion de classe est constituée d'un ensemble d'actes réfléchis, bien ordonnés respectant une planification rigoureuse de la part des enseignants, visant l'instauration d'un climat de travail favorable à l'apprentissage. Gaudreau (2017) précise que la gestion de classe compte cinq grandes dimensions : 1. la gestion des ressources (temporelles, spatiales, matérielles, technologiques et humaines); 2. l'établissement d'attentes claires à l'endroit des élèves; 3. le développement de relations sociales positives; 4. l'obtention de l'attention et de l'engagement des élèves sur l'objet d'apprentissage et, enfin, 5. la gestion des comportements d'indiscipline. C'est à cette dernière dimension que nous nous attardons dans cet article.

\subsection{La gestion des comportements d'indiscipline}

Dans certaines classes, la gestion des comportements d'indiscipline (aussi désignés par l'appellation comportements perturbateurs $(\mathrm{CP})$ ) des élèves occupe jusqu'à $50 \%$ du temps d'enseignement (Gaudreau, 2017). Ceci occasionne beaucoup de frustration chez les enseignants. De façon générale, les écoles primaires et secondaires d'Amérique du Nord comptent environ $15 \%$ d'élèves qui présentent des difficultés comportementales nécessitant l'application d'interventions ciblées et de $3 \%$ à $5 \%$ d'élèves qui présentent des troubles chroniques de comportement exigeant la mise en place d'interventions dirigées (MEESR, 2015). Faute de résultats de recherche, nous ne savons pas si ces proportions sont les mêmes dans les écoles du système d'éducation tunisien.

Les enseignants stagiaires et débutants vivent négativement les épisodes marqués par l'indiscipline et la démotivation des élèves et cela les préoccupe beaucoup (Desbiens, Bali, Spallanzani, Vandercleyen, \& Beaudoin, 2018). D'ailleurs, une recherche réalisée par Habak, Desbiens \& Correa-Molina (2016) rapporte que $50 \%$ des causes de difficultés et d'échecs répertoriées en stage sont liées aux compétences à piloter les situations d'enseignement-apprentissage, à gérer la classe et à adapter l'enseignement aux besoins et aux capacités des élèves. Il est clair que lorsque ces difficultés ne trouvent pas de solution viable, elles peuvent mener à l'installation chez les stagiaires d'un sentiment de démotivation face à la tâche enseignante de même qu'à l'érosion progressive de leur sentiment d'auto-efficacité (Amamou, Desbiens, Spallanzani \& Vandercleyen, 2017; Bandura, 2007). Ainsi, lorsque les stagiaires font face à des comportements d'indiscipline, qu'ils ne parviennent pas à contrôler, ils peuvent se sentir dépassés et pris de panique. Différents travaux (Desbiens et al., 2008; Opinel, 2001), indiquent que les difficultés des stagiaires en éducation physique (EP) à gérer leur classe se traduisent par des pertes de contrôle du fonctionnement du groupe d'élèves de même qu'en d'importantes pertes de temps. Ils ont notamment de la difficulté à interrompre les manifestations d'indiscipline tout en maintenant le rythme et l'intégrité des activités en cours (Goyette, Doré, \& Dion, 2000). De surcroît, leurs interventions sont souvent animées par des croyances et des schémas d'actions simplistes, voire déficitaires en matière de gestion de l'indiscipline (Chouinard, 1999; Kearney, 1987). Contrairement à ce qu'a constaté Hodges Kulinna (2008) chez des enseignants d'EP praticiens, Desbiens et al. (2008) ont observé que les cours dirigés par des stagiaires québécois de deuxième et de quatrième année en EP sont caractérisés par un degré élevé de perturbation. En effet, on y relève un taux de $0,82 \mathrm{CP}$ par minute. 
Ces difficultés des stagiaires à gérer l'indiscipline sont associées selon plusieurs travaux de recherches (Atici, 2007; Bali, 2015; Chouinard, 1999; Desbiens et al., 2011; Goyette et al., 2000; Lavay, Henderson, French \& Guthrie, 2012) à des lacunes dans les cursus de formation. Selon ces chercheurs, les enseignants stagiaires sont, de manière générale, insuffisamment formés pour composer de façon stratégique avec la plupart des comportements d'indiscipline manifestés en classe. En effet, malgré l'importance reconnue, de développer chez eux des compétences visant à prévenir et à réagir efficacement aux comportements d'indiscipline (Maddeh, Desbiens, \& Souissi, 2018 ; Freeman et al., 2014 ; Desbiens et al., 2011), le temps consacré à l'apprentissage de principes de gestion des comportements difficiles en formation initiale à l'enseignement, dans 11 pays étudiés ne correspond qu'à une proportion de 3,4\% du temps moyen consacré à la formation (Gaudreau, 2017). Dans leur étude, Freeman, Simonsen, Briere \& MacSuga-Gage (2013) rapportent que si la plupart des programmes de formation initiale à l'enseignement examinés comportaient au moins un cours de gestion de classe, seulement $60 \%$ d'entre eux présentaient des contenus à caractère scientifique alors que moins de la moitié de ces programmes proposaient des pratiques fondées sur des évidences.

Selon des travaux de recherche tunisiens (Maddeh, Hermessi, Bennour, \& Souissi, 2015 a; Maddeh, Bennour, \& Souissi, 2015 b), la formation préparatoire à l'enseignement de l'EP ne compte aucun cours spécifiquement dédié à la prévention ainsi qu'à la gestion des comportements perturbateurs ni de cours de gestion de classe à proprement parler. Devant ce constat, il est apparu pertinent d'élaborer et de mettre en œuvre un programme de formation en prévention et en gestion de l'indiscipline complémentaire à la formation initiale régulière destinée à des enseignants stagiaires tunisiens en EP et sportive (ÉPS) et de documenter ses effets. Cet article poursuit deux objectifs :

1- Décrire et analyser les effets d'une formation complémentaire sur la manière dont les stagiaires en enseignement de l'ÉPS s'y prennent pour gérer les comportements d'indiscipline des élèves ;

2- $\quad$ Comparer la fréquence des épisodes de gestion préventive, réactive et organisationnelle entre le groupe expérimental $\left(\mathrm{Gr}_{\mathrm{Exp}}\right)$ et le groupe témoin $\left(\mathrm{Gr}_{\mathrm{Tém}}\right)$ en fonction des trois moments de prélèvements pendant l'année scolaire (au début, au milieu et à la fin du stage).

\subsection{Cadre théorique : les trois modes de gestion de l'indiscipline selon Siber (2001)}

Gaudreau (2017; p. 119) définit l'indiscipline comme : «Tout comportement de distraction, de désengagement ou de désobéissance amenant l'enseignant à interrompre son enseignement, à réprimander les élèves, à rétablir l'ordre en classe ou à restructurer la tâche avant de poursuivre. » À cela, Sieber (2001, p. 43) ajoute que les comportements d'indiscipline sont ou non perçus comme une menace à l'instruction à un moment particulier de l'activité de la classe. Ceci suggère qu'une partie de l'apprentissage de la gestion de classe et de la gestion des comportements perturbateurs devrait consister à aider l'enseignant stagiaire non seulement à intervenir face à l'indiscipline, mais surtout à développer ses capacités d'interprète des situations de classe qui se dessinent sous l'influence de ses actions et de celles de ses élèves (Desbiens, Lanoue, Turcotte, Tourigny \& Spallanzani, 2009; Tardif \& Lessard, 1999).

Afin d'analyser et d'interpréter les interventions des enseignants stagiaires en classe d'EP face aux comportements d'indiscipline des élèves, nous nous sommes appuyés sur le modèle développé par Sieber (2001) et dont une description a déjà été fournie dans une de nos études antérieures (Maddeh, Desbiens \& Souissi, 2018). Parmi les différentes dimensions que compte ce modèle, Sieber (2001) met de l'avant trois modes de gestion de l'indiscipline (voir annexe 1) : 1) la gestion préventive, 2) la gestion organisationnelle et 3 ) la gestion réactive.

La gestion préventive est considérée comme une prévention directe de l'apparition de l'indiscipline dans le système classe. Sieber (2001) avance que celle-ci se compose de deux structures. La première touche les intermèdes préventifs :1) la gestion collective avec l'ensemble du groupeclasse, 2) la gestion individualisée avec l'élève jugé comme indiscipliné, et 3) la gestion en collaboration avec les parents de l'élève. Ce dernier élément n'est pas intégré à l'enseignement, mais 
consiste en des discussions à vocation d'éducation générale et sociale. Ces interventions n'ont pas une visée réprobatrice, mais viennent soutenir l'identification de nouvelles stratégies pour l'amélioration de l'équilibre écologique de la séance. La deuxième concerne la gestion préventive intégrée à l'enseignement et consiste en l'installation d'une panoplie de règles sociales que chacun des élèves doit respecter. En d'autres termes, celle-ci réside dans un système de règles énoncées généralement par l'enseignant au début de l'année scolaire représentant le volet disciplinaire du contrat didactique entre l'enseignant et ses élèves. Gaudreau (2019) appelle ce type de pratique par l'intervention universelle stipulante de l'enseignant des directives claires et directes avec un ton de voix calme, une supervision active (Desbiens, 2002) se basant sur des déplacements fréquents entre les élèves et un usage de rétroactions positives fréquentes dans une perspective de renforcement des bons comportements.

Pour Sieber (2001), la gestion organisationnelle réfère au contrôle exercé par les enseignants stagiaires sur trois dimensions essentielles des programmes d'action conçus et mis en œuvre dans un cours d'EP : la gestion de l'environnement d'enseignement-apprentissage, la gestion du placement des élèves et la gestion du temps. Selon les objets traités, et les configurations de travail adoptées, chaque cours d'EP est constitué d'un nombre variable de programmes d'action. Un programme d'action est un segment de temps pendant lequel l'enseignant et les élèves interagissent pour accomplir certaines tâches à caractère scolaire. Tout programme d'action a une direction et un rythme qui mènent vers la réalisation de tâches spécifiques. Il implique un contenu, un arrangement des personnes, du matériel et de l'environnement d'apprentissage (Leriche, Desbiens, Amade-Escot \& Tinning, 2016). La gestion de l'environnement d'enseignement-apprentissage concerne la manière dont l'enseignant stagiaire aménage l'environnement physique de son plateau de travail selon la nature des programmes d'action prévus. La gestion du placement des élèves sur le plateau concerne l'organisation sociale de la classe en considération des spécificités des programmes d'action mis en œuvre. Enfin, la gestion du temps touche la planification et l'ajustement de la durée des différents segments des séances qu'il s'agisse de l'accueil, des transitions, des explications, des temps de jeu, des moments de bilan, etc. Plusieurs chercheurs (Doyle, 1986; Desbiens et al., 2008; Kounin, 1970; Sieber, 2001) rapportent que les enseignants qui négligent de planifier la durée des différents segments d'une séance risquent davantage d'être confrontés à l'apparition des comportements d'indiscipline.

Pour terminer, Sieber (2001) subdivise la gestion réactive en cinq dimensions ou formes génériques : la remontrance, la clarification, la gestion indirecte, la diversion et l'ignorance que l'on peut qualifier de stratégiques (Gaudreau 2017) (annexe 1). Ces formes génériques peuvent être utilisées par les enseignants selon la nature et l'intensité des comportements d'indiscipline qui surgissent lors des cours, mais aussi selon les circonstances ainsi qu'en considération des caractéristiques et des antécédents des élèves concernés. La gestion réactive suppose alors une gestion différenciée des comportements d'indiscipline qui, on le conçoit peut être plus difficile pour l'enseignant stagiaire ou pour l'enseignant remplaçant du fait qu'ils ne peuvent pas s'appuyer aussi fortement que l'enseignant régulier sur la dimension d'historicité (Doyle, 1986). Cet aspect différencié de la gestion réactive consiste dans le fait de prendre en considération lors du choix de l'intervention le degré d'intrusion du comportement indiscipliné, son émetteur, le contexte pour ensuite peser les conséquences du choix de réaliser ou non une fracture ou une rupture de l'enseignement.

Les réactions impliquant la remontrance, la clarification et la gestion indirecte supposent de créer une fracture de la continuité pédagogique, ce que les tenants de l'approche de l'écologie de la classe (Leriche et al., 2016) appellent une interruption du vecteur de l'instruction (le vecteur 1). Chaque interruption constitue un risque que les enseignants stagiaires doivent mesurer avec soin, car il suppose l'arrêt de processus d'engagement dont le redémarrage demeure toujours incertain (Doyle, 1986). Voilà pourquoi des enseignants stagiaires peuvent être tentés de privilégier la continuité du vecteur d'instruction en adoptant des techniques d'intervention moins intrusives comme la diversion ou l'ignorance stratégique (annexe 1) dans l'espoir de détourner l'attention des élèves du ou des comportements d'indiscipline manifestés tout en préservant leur engagement dans les situations d'apprentissage (Maddeh et al., 2018 ; Sieber, 2001). 


\section{METHODOLOGIE}

Dans cette partie, nous présentons le programme d'intervention conçu pour les finalités de cette recherche. Le protocole quasi expérimental adopté, les participants recrutés, la démarche méthodologique, ainsi que le matériel utilisé pour la collecte des données seront ensuite exposés. Nous terminons par l'explication des procédures du traitement et de l'analyse statistique des données.

\subsection{Conception et déroulement du programme d'intervention}

Le programme d'intervention auquel ont été soumis les stagiaires du groupe expérimental $\left(\mathrm{Gr}_{\mathrm{Exp}}\right)$ suit une démarche longitudinale (Figure 1). Il s'étend sur toute la durée de la dernière année du programme de formation initiale des enseignants d'EP de l'ISSEP Ksar Said (Tunisie). Il est composé de deux volets : un premier à caractère théorique suivi d'un volet à caractère pratique. Le programme d'intervention a pour thème principal la prévention et la gestion des comportements perturbateurs lors des cours d'EP.

Le volet de formation à caractère théorique (Maddeh et al., 2018) s'appuie sur le modèle de Sieber (2001). Il compte cinq activités de formation. Les trois premières ont été données sous la forme d'exposés d'une durée de deux heures chacune. Dans la quatrième activité, des séquences vidéo traitant des différents types d'évènements disciplinaires et tirées de vidéos des cours de stagiaires de l'année précédente ont été présentées aux enseignants stagiaires. Un arrêt de la projection a été réalisé lorsque ces derniers repéraient une des catégories d'évènements disciplinaires. Une analyse systématique traitant de l'évènement ciblé était réalisée immédiatement par les stagiaires sous forme d'une discussion animée par le formateur.

La cinquième activité a été réalisée individuellement. Lors des séances de rétroaction post intervention hebdomadaire, deux extraits vidéos de cinq minutes chacun (choisis par le formateur sur la base d'une forte concentration de faits d'indiscipline) tirés de la séance qu'ils venaient d'animer ont été présentés aux enseignants stagiaires du $\mathrm{Gr}_{\text {Exp. }}$. Un arrêt était réalisé à chaque fois qu'un stagiaire détectait un évènement disciplinaire. Le même stagiaire devait alors analyser verbalement l'évènement soulevé puis il recevait les rétroactions correctives et les renforcements appropriés de la part du formateur. Les trois premières formations ont été données dans l'établissement universitaire des stagiaires (ISSEP de Tunis) avant le début de leur stage (les deux dernières semaines de septembre), alors que les deux dernières se sont déroulées lors des séances de retro action dans le milieu d'accueil des stagiaires (Maddeh et al., 2018).

Dans le deuxième volet pratique de la formation, il s'agissait de développer la capacité des stagiaires du $\mathrm{Gr}_{\text {Exp }}$ d'analyser et de résoudre des problèmes d'indiscipline en classe. Ce volet fait une utilisation extensive de la vidéo in situ à travers cinq types d'intervention. Ces derniers étaient étalés sur la totalité de l'année selon un calendrier bien déterminé (Maddeh et al., 2018) et d'une façon répétitive lors des séances de feed-back réalisées à la fin de la journée du stage d'enseignement pratique dans l'établissement qui accueillait les stagiaires. Ces interventions avaient une visée d'analyser les situations réelles d'enseignement en instaurant une dialectique entre la réflexion issue des formations théoriques et la pratique réalisée au lors de l'enseignement.

Cette dialectique avait pour vocation de rendre les stagiaires capables de transcrire soit verbalement ou visuellement les différentes catégories des CP susceptibles d'apparaitre lors des cours d'enseignement, ainsi que les différentes formes de gestion réalisées pour les gérer. La transcription verbale s'est effectuée au travers des discussions d'analyse portant sur des séquences choisies par le formateur et laissant l'alternative aux stagiaires de déterminer verbalement la nature des CP et la forme de la gestion qui a été émise. Quant à la transcription visuelle, celle-ci s'est déroulée en utilisant la grille d'observation de Siber (2001) en analysant soit des séquences choisies par le formateur soit de vidéos se rapportant aux situations réelles d'enseignement issu du stage pratique. L'objectif de cette intervention (Maddeh et al., 2018) consiste à repérer, identifier et nommer les différents faits indisciplinaires ainsi que les réactions émises par le stagiaire. 
En résumé, notre programme de formation (les formations théoriques et les interventions pratiques) a été réalisé auprès des stagiaires du $\mathrm{Gr}_{\text {Exp }}$ par le chercheur lui-même (Figure 1). Celui-ci agit aussi comme un superviseur non responsable de l'évaluation du stage pratique des enseignants stagiaires participant à cette étude. Le schéma ci-dessous présente le déroulement des différentes composantes de notre formation complémentaire qui s'est étalée sur une année entière.

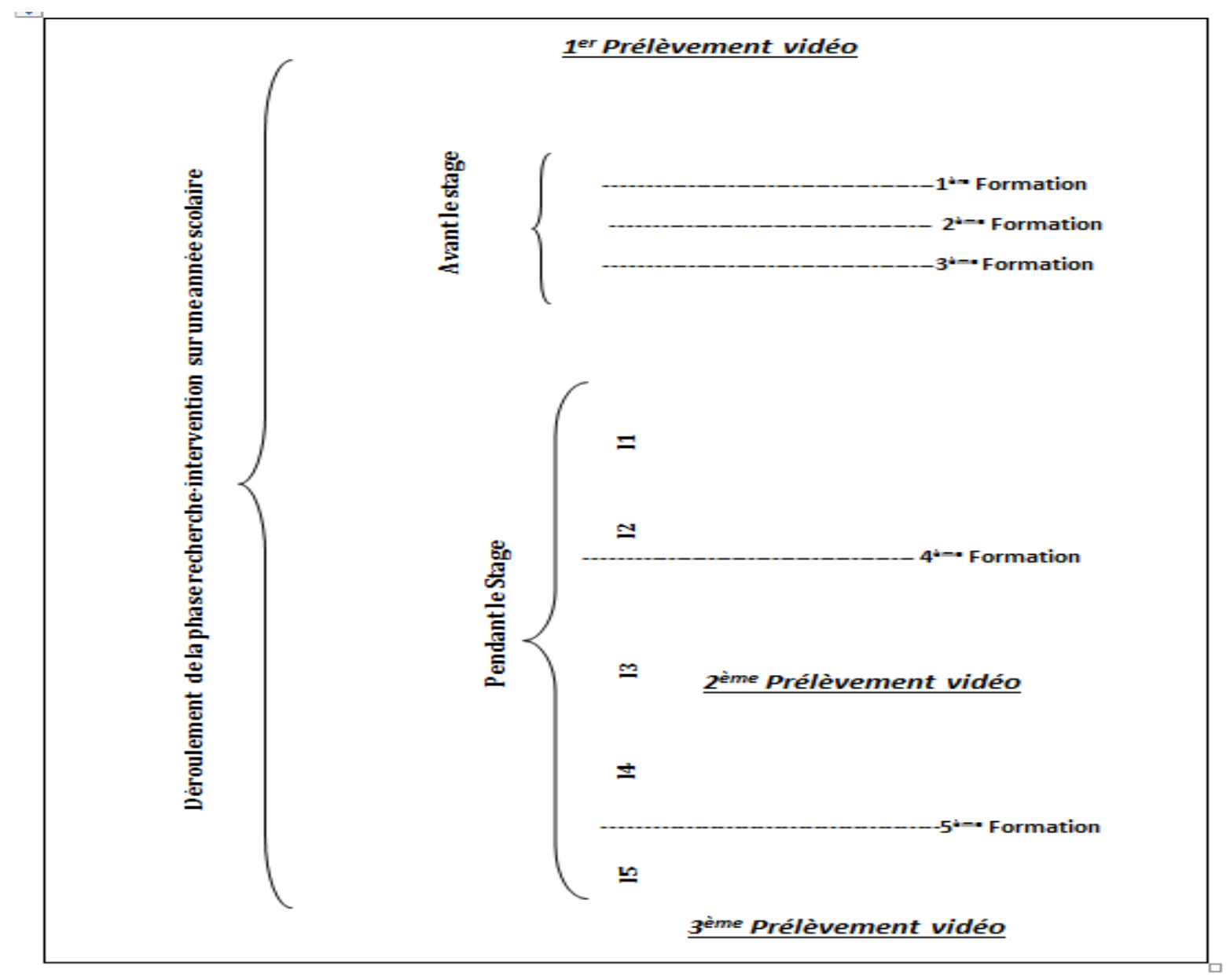

Figure 1: Articulation temporelle des démarches de formation à la gestion des comportements perturbateurs et de collecte des données

\subsection{Participants}

L'échantillon était constitué de dix enseignants stagiaires d'EP tunisiens de sexe masculin choisis sur la base du volontariat et de leur accessibilité sur le plan géographique. Pour ces raisons, il s'agit d'un échantillon non probabiliste de convenance. Cinq stagiaires formant le $\mathrm{Gr}_{\mathrm{Exp}}$ étaient affectés en stage dans un même établissement. Les cinq autres formant le groupe témoin $\left(\mathrm{Gr}_{T e ́ m}\right)$ étaient affectés dans un autre établissement. Les deux établissements scolaires étaient localisés dans un même quartier qualifié de difficile pour l'enseignement au regard du ministère de l'enseignement Tunisien. Les établissements accueillaient la même catégorie d'élèves issus de ce même milieu.

Tous les participants étaient des finissants inscrits en troisième (dernière) année du programme de licence fondamentale en enseignement de l'EP. Ils réalisaient leur stage dans un milieu d'enseignement secondaire. Les stages étaient d'une durée de trente semaines, à raison de quatre heures de pratique pour chacune d'elles, soit un total de 120 heures de pratique annuelle. Chacun des 
épisodes de quatre heures correspond à un bloc de quatre leçons de 55 minutes traitant toujours avec les mêmes groupes d'élèves.

Tous les participants ont préalablement été informés de nos visées de l'étude ainsi que des dispositions prises pour la préservation de leur anonymat ainsi que la confidentialité des données recueillies. Un total de 170 élèves (Mâge : 13,22 $\pm 0,35)$ du secondaire dont $110(64,7 \%)$ sont de sexe masculin et $60(35,3 \%)$ de sexe féminin ont participé à cette étude avec une moyenne de 34 élèves par classe. Ces derniers étaient engagés dans deux activités de sports collectifs (handball et basketball).

\subsection{Collecte des données}

La collecte des données audiovisuelles a été réalisée au moyen de deux caméscopes (Model; Handcam $4 \mathrm{~K}$ ) avec un projecteur intégré et un micro sans fils avec un récepteur émetteur DJ (VHF 10HL F4 Micro H.F) dont la portée était d'un rayon de quatre-vingts mètres. La collecte s'est effectuée sur une durée d'une année à raison de trois captures par participant (30 séances de 55 minutes au total) (P1 : début d'année lors du mois de septembre, P2 : milieu d'année lors du mois de janvier, P3 : fin d'année lors du mois de juin). Deux chercheurs ont été formés aux procédures et aux techniques de la captation vidéo. Les caméras étaient positionnées en diagonale, l'une se focalisant sur l'enseignant stagiaire lors de l'enseignement en étant connectée avec le micro sans fils pour capter ses interventions verbales, l'autre étant destinée à filmer l'ensemble du plateau de travail avec les élèves (objectif grandangle). Chacun des trois épisodes de captation a été effectué sur une durée de deux semaines successives. Durant la première semaine, le groupe expérimental a été filmé. Le groupe témoin a été filmé durant la deuxième semaine. Dans une perspective d'atténuation de l'impact de l'observateur et des caméras sur les élèves, les deux chercheurs ont été impliqués préalablement à la collecte des données dans une semaine de familiarisation en réalisant des enregistrements d'essai avec les classes concernées.

\subsection{Traitement et analyse des données}

\subsubsection{Traitement des données}

L'observation vidéoscopique événementielle en différé a menée au codage des comportements d'indiscipline à l'aide de grille d'observation ainsi qu'en leur regroupement selon les trois catégories (prévention, organisation et réaction) proposées par Sieber (2001). Ce codage des données ainsi que leur transposition sur support informatique a été réalisé par un analyste formé au modèle de gestion de l'indiscipline de Sieber (2001) en collaboration avec deux autres chercheurs ayant aussi été formés à ce protocole.

Le chercheur a réalisé le traitement des données informatisées au moyen d'une matrice correspondant aux trois différentes formes de gestion par le biais de la grille d'observation de Sieber (2001) qui permet la saisie des données de recherche. Pour être comptabilisé, l'évènement disciplinaire doit avoir une durée comprise entre 5 et 30 secondes (Desbiens et al., 2011). La forme de la gestion (préventive, organisationnelle ou réactive) utilisée par le stagiaire face à chacun des évènements lui est associée et est enregistrée avec son code correspondant sur la grille d'observation. L'inscription de ce dernier se fera dans le cas où l'incident est vu par le stagiaire observé. Dans le cas inverse, c'est-à-dire si ce dernier n'a pas vu le CP et qu'il n'a pas réagi, on comptabilise un fait de " gestion nulle ».

\subsubsection{Analyse des données}

L'analyse des données a été réalisée à l'aide de techniques statistiques non paramétriques en raison de la petite taille de l'échantillon $(\mathrm{n}=10)$. L'analyse descriptive consiste tout d'abord en un dénombrement des fréquences absolues (f) et relatives ( $\mathrm{f} \%$ ) des comportements selon leur ordre 
d'apparition suivant la grille adoptée à partir du modèle de gestion de l'indiscipline de Sieber (2001). Ces données ont été analysées sur le plan statistique avec le logiciel SPSS de la version 24.

Nous avons procédé à des comparaisons intra et inter-groupes selon les trois moments de prélèvement identifiés plus haut en vue d'apprécier l'évolution du degré d'indiscipline dans les classes dirigées par les stagiaires des groupes expérimentaux $\left(\mathrm{Gr}_{\mathrm{Exp}}\right)$ et témoins $\left(\mathrm{Gr}_{\mathrm{Tém}}\right)$, mais aussi celle des pratiques utilisées par ceux-ci durant les enseignements pour prévenir ou réagir à l'indiscipline des élèves.

Les comparaisons entre le $\mathrm{Gr}_{\mathrm{Exp}}$ et le $\mathrm{Gr}_{\text {Tém }}$ d'un épisode de collecte de données à un autre ont été effectuées à l'aide du test de Mann-Whitney. Pour les comparaisons groupe par groupe entre les différents moments de prélèvements, le test de Friedman a été utilisé (Howel, 2008) suivi du test de Wilcoxon (Howel, 2008) utilisé comme test post-hoc. Dans ces cas précis, le caractère significatif ou non des résultats a été apprécié après avoir apporté une correction pour comparaisons multiples à l'aide du test de Bonferroni $(\alpha=, 05 / 3=, 016)$.

\subsection{Fidélité inter juges}

Deux chercheurs ont été formés pour assurer la fidélité de la procédure de codification des données conformément à la grille d'observation issue du modèle de Sieber (2001). La confrontation de leurs perceptions pour les différents résultats suite à la codification d'une même séance d'enseignement choisie au hasard parmi les 30 séances filmées, a donné une concordance moyenne de $87 \%$. En effet, pour la variable de la détection des CP, la moyenne a été de $89 \%$ alors que celle pour la détection des trois formes de la gestion des comportements perturbateurs a été de $86 \%$ (85\% pour la gestion réactive, $87 \%$ pour la gestion organisationnelle et $86 \%$ pour la gestion préventive). Selon Fortin (2010), ces degrés de concordance inter-codeurs sont considérés satisfaisants. 


\section{RÉSULTATS}

Dans cette section nous présentons les résultats de la recherche. Cette présentation s'effectuera en deux temps. D'abord, il sera question de comparer les résultats obtenus groupe par groupe pour chacune des trois formes de gestion, et ce dans une perspective évolutive, c'est-à-dire en tenant compte de trois moments précis de collecte des données. Ensuite, nous comparerons les résultats de chacun des groupes à chacun des moments de collecte des données pour chacune des formes de gestion de l'indiscipline.

\section{1. Évolution des manières d'intervenir face à l'indiscipline des stagiaires des groupes témoin et expérimental}

Le tableau 1 rapporte les résultats décrivant la fréquence d'utilisation des comportements d'intervention à caractère préventif, organisationnel et réactif par les stagiaires des groupes expérimentaux $\left(\mathrm{Gr}_{\text {Exp. }}\right.$.) et témoin $\left(\mathrm{Gr}_{\text {Tém. }}\right.$.) au tout début de l'étude c'est-à-dire au premier prélèvement.

Tableau 1 : Fréquence d'utilisation des différentes catégories de comportements de gestion de l'indiscipline par les stagiaires des groupes expérimentaux et témoin au début de l'étude

\begin{tabular}{lcccccccc}
\hline & \multicolumn{3}{c}{ Groupe expérimental $\left(\mathbf{G r}_{\text {Exp. }}\right)$} & \multicolumn{3}{c}{ Groupe témoin (Gr Tém.) } \\
\hline \multirow{2}{*}{ GP } & F & F(\%) & M \pm É-C & Md & F & F(\%) & M \pm É-C & Md \\
\cline { 2 - 8 } GO & 18 & 7,35 & $3,6 \pm 1,48$ & 4 & 21 & 8,68 & $4,2 \pm 1,78$ & 4 \\
GR & 96 & 39,18 & $19,2 \pm 1,09$ & 19 & 98 & 40,49 & $19,6 \pm 1,51$ & 19 \\
Total & 131 & 53,47 & $26,2 \pm 2,38$ & 25 & 123 & 50,83 & $24,6 \pm 1,51$ & 24 \\
\hline
\end{tabular}

Ces résultats descriptifs indiquent que tant chez les stagiaires du groupe expérimental que chez ceux du groupe contrôle, les interventions de gestion préventive sont les moins fréquentes suivies des interventions de gestion organisationnelle et, finalement, des interventions de gestion réactive. Notons, en outre, que pour chaque catégorie de stagiaires, les fréquences d'utilisation rapportées sont à peu près de même magnitude.

Le tableau 2 rapporte des résultats décrivant l'utilisation des comportements d'intervention à caractère préventif, organisationnel et réactif par les stagiaires des $\mathrm{Gr}_{\text {Exp }}$. et $\mathrm{Gr}_{\text {Tém., }}$, au milieu de l'étude c'est-à-dire au second prélèvement des données.

Tableau 2 : Fréquence d'utilisation des différentes catégories de comportements de gestion de l'indiscipline par les stagiaires des groupes expérimentaux et témoin au milieu de l'étude

\begin{tabular}{lcccccccc}
\hline & \multicolumn{3}{c}{ Groupe expérimental $\left(\mathbf{G r}_{\text {Exp. }}\right)$} & \multicolumn{3}{c}{ Groupe témoin (GrTém.) } \\
\hline \multirow{2}{*}{ GP } & $\mathbf{F}$ & $\mathbf{F}(\%)$ & $\mathbf{M} \pm \mathbf{E}-\mathbf{C}$ & $\mathbf{M d}$ & $\mathbf{F}$ & $\mathbf{F}(\%)$ & $\mathbf{M} \pm \mathbf{E}-\mathbf{C}$ & Md \\
\cline { 2 - 8 } & 45 & 16,67 & $9 \pm 2,64$ & 9 & 22 & 8,66 & $4,4 \pm 1,51$ & 4 \\
GO & 107 & 39,63 & $21,4 \pm 1,14$ & 21 & 110 & 43,31 & $22 \pm 2,12$ & 22 \\
GR & 118 & 43,70 & $23,6 \pm 1,51$ & 18 & 122 & 48,03 & $24,4 \pm 2,07$ & 24 \\
Total & 270 & 100 & ---- & ---- & 254 & 100 & ---- & ---- \\
\hline
\end{tabular}


Comme dans le cas des résultats obtenus lors du premier prélèvement, les interventions de gestion préventive demeurent les moins utilisées suivies des interventions de gestion organisationnelle et des interventions de gestion réactive. Toutefois, du côté des stagiaires du groupe expérimental, une réduction marquée du nombre d'interventions à caractère réactif $(-9,77 \%)$ est notée au profit des interventions à caractère préventif $(+9,32 \%)$ alors que les comportements de gestion organisationnelle demeurent relativement stables.

Le tableau 3 rapporte des résultats décrivant l'utilisation des comportements d'intervention à caractère préventif, organisationnel et réactif par les stagiaires des $\mathrm{Gr}_{\text {Exp. }}$. et $\mathrm{Gr}_{\text {Tém. }}$ à la fin de l'étude c'est-à-dire au troisième et dernier prélèvement.

Tableau 3 : Fréquence d'utilisation des différentes catégories de comportements de gestion de l'indiscipline par les stagiaires des groupes expérimentaux et témoin à la fin de l'étude

\begin{tabular}{lcccccccc}
\hline & \multicolumn{3}{c}{ Groupe expérimental $\left(\mathbf{G r}_{\text {Exp. }}\right)$} & \multicolumn{3}{c}{ Groupe témoin $\left(\mathbf{G r}_{\text {Tém. }}\right)$} \\
\cline { 2 - 8 } & $\mathbf{F}$ & $\mathbf{F}(\%)$ & $\mathbf{M} \pm \mathbf{E}-\mathbf{C}$ & $\mathbf{M d}$ & $\mathbf{F}$ & $\mathbf{F}(\%)$ & $\mathbf{M} \pm \mathbf{E}-\mathbf{C}$ & Md \\
\hline GP & 69 & 23,16 & $13,8 \pm 3,42$ & 14 & 26 & 9,93 & $5,2 \pm 1,09$ & 5 \\
GO & 137 & 45,97 & $27,4 \pm 1,67$ & 27 & 121 & 46,18 & $24,2 \pm 2,77$ & 25 \\
GR & 92 & 30,87 & $18,4 \pm 1,67$ & 24 & 115 & 43,89 & $23 \pm 2,54$ & 23 \\
Total & 298 & 100 & ---- & ---- & 262 & 100 & $-\cdots$ & ---- \\
\hline
\end{tabular}

Les résultats présentés dans ce tableau montrent clairement que le patron d'intervention des stagiaires des deux groupes s'est modifié depuis le début et le milieu du stage. Tant du côté des stagiaires du groupe expérimental que chez ceux du groupe témoin, ce sont les interventions de gestion organisationnelle qui sont devenues les plus fréquentes tant en termes absolus que relatifs suivies des interventions de gestion réactive et préventive. Chez les stagiaires du premier groupe, une diminution de $12,83 \%$ du nombre d'interventions de gestion réactive est enregistrée alors que des augmentations de $6,34 \%$ et de $6,49 \%$ sont constatées dans la fréquence des interventions de gestion organisationnelle et préventive respectivement. Les modifications remarquées chez les stagiaires du second groupe concernent plus spécifiquement les interventions de gestion réactive $(-4,14 \%)$ et organisationnelle $(+2,87 \%)$, mais de manière négligeable les interventions à caractère préventif $(+1,27 \%)$.

Les résultats présentés dans le tableau 4 confirment que les stagiaires des $\mathrm{Gr}_{\text {Exp }}$ et $\mathrm{Gr}_{\text {Tém }}$ utilisent différemment les comportements d'intervention regroupés sous chacun des trois modes de gestion de l'indiscipline en fonction des moments de collecte des données. Du côté des stagiaires du groupe expérimental, des différences statistiquement significatives sont constatées pour chacun d'eux alors que chez les stagiaires du groupe témoin, deux différences significatives sont rapportées qu'en ce qui concerne les comportements de gestion organisationnelle et de gestion réactive. 
Tableau 4 : Résultats du test de Friedman concernant la fréquence des comportements regroupés sous chacun des trois modes de gestion de l'indiscipline par les stagiaires des groupes expérimentaux et témoin en fonction des moments de prélèvement

\begin{tabular}{lcc}
\hline $\begin{array}{c}\text { Les modes de } \\
\text { gestion de } \\
\text { l'indiscipline }\end{array}$ & Groupe expérimental $\left(\mathbf{G r}_{\text {Exp. }}\right)$ & Groupe témoin $\left(\mathbf{G r}_{\text {Tém. }}\right)$ \\
\hline $\begin{array}{l}\text { Gestion } \\
\text { préventive (GP) }\end{array}$ & $X^{2}=10,00$ & \\
& $\mathrm{ddl}=2$ & $X^{2}=1,200$ \\
Gestion & $\mathrm{p}=, 007$ & $\mathrm{ddl}=2$ \\
organisationnelle & $X^{2}=10,00$ & $\mathrm{p}=, 549$ \\
(GO) & $\mathrm{ddl}=2$ & $X^{2}=9,579$ \\
& $\mathrm{p}=, 007$ & $\mathrm{ddl}=2$ \\
Gestion réactive & $X^{2}=8,316$ & $\mathrm{p}=, 008$ \\
(GR) & $\mathrm{ddl}=2$ & $X^{2}=7,684$ \\
& $\mathrm{p}=, 016$ & $\mathrm{ddl}=2$ \\
\hline
\end{tabular}

Les résultats présentés dans le tableau 5 permettent de comparer plus précisément l'évolution de la médiane de la fréquence d'utilisation des comportements relevant des différents modes de gestion de l'indiscipline par les stagiaires des $\mathrm{Gr}_{\mathrm{Exp}}$ et $\mathrm{Gr}_{\text {Tém }}$ entre les différents moments de collecte des données. Rappelons qu'en raison des comparaisons multiples effectuées à l'aide du test de Wilcoxon (W) utilisé ici comme test post hoc, un ajustement de Bonferroni a été réalisé. Ainsi, pour considérer un résultat significatif, alpha doit être $\leq, 016$.

Tableau 5 : Résultats de la comparaison de la fréquence d'utilisation des différents modes de gestion de l'indiscipline par les stagiaires des groupes expérimentaux et témoin en fonction des moments de prélèvement

\begin{tabular}{lcccccc}
\hline \multicolumn{1}{c}{$\begin{array}{c}\text { Les modes de } \\
\text { gestion }\end{array}$} & \multicolumn{3}{c}{ Groupe expérimental $\left(\mathbf{G r}_{\text {Exp }}\right)$} & \multicolumn{2}{c}{ Groupe témoin $\left(\mathbf{G r}_{\text {Tém }}\right)$} \\
\cline { 2 - 7 } & $\mathrm{P} 1-\mathrm{P} 2$ & $\mathrm{P} 2-\mathrm{P} 3$ & $\mathrm{P} 1-\mathrm{P} 3$ & $\mathrm{P} 1-\mathrm{P} 2$ & $\mathrm{P} 2-\mathrm{P} 3$ & $\mathrm{P} 1-\mathrm{P} 3$ \\
\hline Gestion & $z=-2,032$ & $z=-2,023$ & $z=-2,060$ & $z=-, 272$ & $z=-, 876$ & $z=-, 921$ \\
préventive & $\mathrm{p}=, 042$ & $\mathrm{p}=, 043$ & $\mathrm{p}=, 039$ & $\mathrm{p}=, 785$ & $\mathrm{p}=, 414$ & $\mathrm{p}=, 357$ \\
\hline Gestion & $z=-2,032$ & $z=-2,023$ & $z=-2,023$ & $z=-2,032$ & $z=-1,841$ & $z=-2,023$ \\
organisationnelle & $\mathrm{p}=, 042$ & $\mathrm{p}=, 043$ & $\mathrm{p}=, 043$ & $\mathrm{p}=, 042$ & $\mathrm{p}=, 066$ & $\mathrm{p}=, 043$ \\
\hline Gestion réactive & $z=-1,473$ & $z=-2,041$ & $z=-2,023$ & $z=-1,826$ & $z=-, 816$ & $z=-2,032$ \\
& $\mathrm{p}=, 141$ & $\mathrm{p}=, 041$ & $\mathrm{p}=, 043$ & $\mathrm{p}=, 068$ & $\mathrm{p}=, 444$ & $\mathrm{p}=, 042$ \\
\hline
\end{tabular}

L'examen du tableau 5 permet de constater que lorsqu'on compare les résultats médians obtenus par les stagiaires du groupe expérimental et ceux du groupe témoin, aucun d'eux n'est statistiquement significatif au seuil ajusté de 0,016 , c'est-à-dire une fois l'ajustement de Bonferroni pris en compte. Nous concluons que l'évolution de la fréquence d'utilisation des comportements de gestion préventive, de gestion organisationnelle et de gestion réactive par les stagiaires des deux groupes entre le premier et le dernier moment de collecte des données n'est pas statistiquement marquée. Toutefois, à titre indicatif, 11 des 18 résultats présentés dans le tableau 5 seraient significatifs si $\alpha$ avait pour seuil la valeur de, 05 . En outre, six des sept valeurs non significatives se retrouvent du côté des stagiaires du groupe témoin alors qu'une seule se retrouve du côté des stagiaires du groupe expérimental ce qui suggère que le programme d'intervention a engendré des effets qui sont demeurés toutefois sous le seuil de signification ajusté. 


\subsection{Comparaison de la façon dont les stagiaires des groupes témoin et expérimental} interviennent face à l'indiscipline à chacun des trois moments de collecte des données.

D'une manière globale, c'est-à-dire en réalisant un examen comparatif du total des fréquences obtenues à chacun des moments de collecte des données pour chacune des formes d'intervention face à l'indiscipline, il ressort que les stagiaires du groupe expérimental ont réalisé significativement plus d'interventions à visées préventive et organisationnelle que les stagiaires du groupe témoin. De son côté, le nombre d'interventions à visée réactive utilisé par les stagiaires des deux groupes n'est pas différent sur le plan statistique.

Tableau 6 : Comparaison de la fréquence totale des interventions réalisées dans chacune des trois formes de gestion de l'indiscipline par les stagiaires des groupes expérimentaux et témoin

\begin{tabular}{lc}
\hline & Total des séances \\
\hline Gestion & GrExp. $>$ GrTém. \\
préventive & $z=-2,627$ \\
& $\mathrm{p}=, 009$ \\
Gestion & $\mathrm{Gr}_{\text {Exp. }}>$ GrTém. \\
organisationnelle & $z=-2,207$ \\
& $\mathrm{p}=, 027$ \\
& $\mathrm{Gr}_{\text {Exp. }}=$ GrTém. \\
& $z=-, 629$ \\
Gestion réactive & $\mathrm{p}=, 530$ \\
& \\
\hline
\end{tabular}

Une seconde analyse, plus approfondie celle-là, compare la fréquence des interventions posées par les stagiaires des deux groupes à chacun des moments de collecte des données. Cette analyse résumée dans le tableau 7 montre d'abord qu'au moment du premier prélèvement des données, aucune différence significative n'est trouvée entre les deux groupes peu importe le type de gestion de l'indiscipline. Ensuite, elle met en évidence qu'au second temps de prélèvement des données, le seul résultat statistiquement significatif favorise les stagiaires du groupe expérimental. Ceux-ci emploient davantage d'interventions préventives que les stagiaires du groupe témoin. Pour terminer, à la fin du stage, au moment du troisième prélèvement des données, les stagiaires du groupe expérimental emploient significativement plus les interventions de gestion préventive et de gestion organisationnelle que ceux du groupe témoin. 
Tableau 7 : Comparaison de la fréquence des interventions réalisées dans chacune des trois formes de gestion de l'indiscipline par les stagiaires des groupes expérimentaux et témoin à chacun des trois moments de collecte des données

\begin{tabular}{|c|c|c|c|}
\hline & T1 & $\mathrm{T} 2$ & $\mathrm{~T} 3$ \\
\hline $\begin{array}{l}\text { Gestion } \\
\text { préventive }\end{array}$ & $\begin{array}{c}\text { Grexp }=\text { GrTém } \\
U=8,5 \\
\mathrm{p}=, 381\end{array}$ & $\begin{array}{c}\text { Grexp }>\text { GrTém } \\
z=-2,619 \\
\mathrm{p}=, 009\end{array}$ & $\begin{array}{c}\text { Grexp }>\text { GrTém } \\
z=-2,619 \\
\mathrm{p}=, 009\end{array}$ \\
\hline $\begin{array}{l}\text { Gestion } \\
\text { organisationnelle }\end{array}$ & $\begin{array}{c}\mathrm{Gr}_{\exp }=\mathrm{Gr}_{\text {Tém }} \\
U=8,0 \\
\mathrm{p}=, 343\end{array}$ & $\begin{array}{c}\mathrm{Gr}_{\text {exp }}=\mathrm{Gr}_{\text {Tém }} \\
U=7,0 \\
\mathrm{p}=, 242\end{array}$ & $\begin{array}{c}\mathrm{Gr}_{\exp }>\mathrm{Gr}_{\text {Tém }} \\
U=2,5 \\
\mathrm{p}=, 036\end{array}$ \\
\hline Gestion réactive & $\begin{array}{c}\mathrm{Gr}_{\mathrm{exp}}=\mathrm{Gr}_{\text {Tém }} \\
U=10,5 \\
\mathrm{p}=, 669\end{array}$ & $\begin{array}{c}\mathrm{Gr}_{\text {exp }}=\mathrm{Gr}_{\text {Tém }} \\
U=4,5 \\
\mathrm{p}=, 092\end{array}$ & $\begin{array}{c}\mathrm{Gr}_{\text {exp }}=\mathrm{Gr}_{\text {Tém }} \\
U=5,0 \\
\mathrm{p}=, 110\end{array}$ \\
\hline
\end{tabular}

Il ressort que les manières d'intervenir des deux groupes ont évolué sensiblement dans la même direction pendant l'année qu'a durée le stage en enseignement de l'EP, mais que les changements sont statistiquement plus prononcés du côté des stagiaires du premier groupe ce qui suggère un effet bénéfique du programme de formation complémentaire offert à ces derniers.

En clair, le stage en lui-même semble avoir suscité des changements appréciables dans la fréquence d'utilisation des comportements d'intervention regroupés dans les trois catégories de la grille (prévention, organisation et réaction) par les stagiaires auxquels se sont ajoutés ceux découlant de l'implantation du dispositif de formation mis à l'essai auprès des stagiaires du groupe expérimental. Ainsi, en bout de ligne, il est possible de constater que les stagiaires des deux groupes se sont développés sensiblement dans la même direction, mais que les seconds ont montré des changements significativement plus importants que les premiers dans leurs manières de prévenir et de s'organiser.

\section{DISCUSSION}

Depuis 1969, des sondages réalisés épisodiquement aux États-Unis rapportent que la gestion des comportements et la motivation des élèves sont des défis importants pour les enseignants de toutes les disciplines (Lavay et al., 2012). Les problèmes de comportements en classe sont l'une des causes d'inconfort et de mal-être au travail des enseignants (Postholm, 2013) et l'une des principales raisons avancées pour expliquer le départ prématuré d'enseignants novices tôt après leur entrée dans la profession. Comme l'affirme Hodges Kulinna (2008), les comportements d'indiscipline des élèves constituent une menace pour la classe et il importe donc de mieux les comprendre, mais aussi d'y réagir de manière appropriée afin de réduire les dérangements, les pertes de temps et ultimement, pour prévenir l'épuisement professionnel.

Cet article consacré à l'étude de la gestion des comportements perturbateurs par des stagiaires en EP en contexte tunisien poursuivait deux objectifs : 1. décrire et analyser les effets d'une formation complémentaire à la formation initiale à l'enseignement sur l'évolution de la fréquence des épisodes de gestion préventive, réactive et organisationnelle; 2. comparer les effets de cette formation sur la fréquence des épisodes de gestion préventive, réactive et organisationnelle constatée chez les stagiaires du groupe expérimental et chez ceux du groupe contrôle au début, au milieu et à la fin du stage.

De manière générale, dans le cas du premier objectif, les résultats obtenus indiquent que la fréquence d'utilisation des comportements de prévention s'accroît entre le début et la fin du stage chez les stagiaires des deux groupes, mais que cette augmentation est nettement plus importante chez ceux du groupe expérimental que chez ceux du groupe témoin $(+383,33 \%$ c. $+23,80 \%)$. De la même 
façon, mais dans une moindre mesure, une augmentation sensible des comportements d'organisation est constatée chez les stagiaires des deux groupes bien que de façon plus marquée chez ceux du groupe expérimental $(+42,71 \%$ c. $+23,47 \%)$. Enfin, on constate chez les stagiaires des deux groupes une diminution du recours aux comportements réactifs. Ce fléchissement est nettement plus marqué chez les stagiaires du groupe expérimental que chez ceux du groupe témoin (- $29,77 \%$ c. - 6,50\%).

Ces résultats préliminaires suggèrent une influence plus grande de la condition expérimentale (stage + dispositif de formation) sur l'utilisation des comportements d'intervention des stagiaires par rapport à la condition de référence (stage uniquement). Les analyses post hoc n'ont cependant pas permis de dégager de différence significative une fois l'ajustement de Bonferroni pris en compte. Toutefois, comme nous l'avons montré à titre indicatif, une majorité des résultats classés non significatifs quand $\alpha<, 05$ se retrouvent du côté des stagiaires du groupe témoin tandis que huit des onze résultats significatifs dans la même condition se retrouvent du côté des stagiaires du groupe expérimental. Ces résultats suggèrent selon nous qu'avec un nombre plus élevé de participants dans les deux groupes nous aurions pu obtenir des différences plus tranchées sur le plan statistique. Ils traduisent, en outre, un changement de perspective des stagiaires du groupe expérimental dans leur façon d'aborder la gestion des comportements perturbateurs ainsi qu'une possible prise de conscience de leur part que l'enseignant a un rôle important à jouer dans la construction d'un climat propice à l'apprentissage en amont de la manifestation des comportements d'indiscipline par l'adoption de comportements visant la prévention et l'organisation.

Dans le cas du second objectif, il ressort que les effets de la formation complémentaire sur la fréquence d'utilisation des comportements d'intervention ne se sont pas fait sentir de manière uniforme. Par exemple, aucune différence significative n'est rapportée entre les stagiaires des deux groupes, à quelque moment que ce soit, concernant l'utilisation des comportements de gestion réactive. Une différence significative en faveur des stagiaires du groupe expérimental n'est constatée qu'en fin de stage pour les comportements de gestion organisationnelle alors que deux différences significatives, toujours au profit des stagiaires du même groupe sont rapportées au milieu et en fin de stage en ce qui a trait à la fréquence d'utilisation des comportements de gestion préventive. Il y a lieu d'examiner de manière plus spécifique le contenu, les modalités et la proportion du temps de formation consacré aux comportements de gestion réactive afin de mieux comprendre pourquoi les stagiaires du groupe expérimental ne se démarquent pas de manière significative de ceux du groupe témoin malgré un écart de $23,27 \%$ quant à la fréquence d'utilisation de ces réactions. Ici encore, on ne peut pas exclure que le faible nombre de participants soit en cause, mais sans doute y a-t-il plus.

Comme Siedentop (1994) l'a déjà bien montré, toutes les conclusions de la recherche sur l'efficacité de l'enseignement s'entendent sur le fait qu'une bonne organisation préventive permet de gagner du temps et de réduire la fréquence des comportements d'indiscipline. Les comportements de gestion préventive et de gestion organisationnelle servent à jeter les bases de la nécessaire coopération entre stagiaires et élèves. Contrairement aux comportements de gestion réactive qui sont émis en réponse à des comportements émergents de la part des élèves, les gestions préventive et organisationnelle tels que l'établissement de règles et de routines, l'utilisation de signaux pour rétablir l'ordre, l'aménagement du plateau de travail, l'organisation socio-spatiale du groupe et la gestion du temps se repèrent, s'apprécient et se contrôlent autrement (Desbiens et al., 2009). Ils sont planifiés à l'avance et font partie des conventions que l'on établit en tout premier lieu lorsque l'on prend en charge un groupe d'élèves (Archambault et Chouinard, 2009; Gaudreau, 2017). Ils correspondent à ce que Gaudreau (2019) appelle des interventions universelles qui participent de la prévention primaire des manifestations d'indiscipline. Nous faisons donc l'hypothèse que les stagiaires du groupe expérimental ont appris par la conjonction du stage et de la formation complémentaire à les utiliser davantage au profit de la création d'un climat favorable à l'apprentissage. 


\section{CONCLUSION}

En conclusion, cette étude indique qu'une formation en matière de prévention et de gestion de l'indiscipline qui est complémentaire à la formation initiale peut amener des stagiaires d'EP tunisiens à modifier significativement leurs manières d'intervenir face aux comportements d'indiscipline des élèves. Le spectre des préoccupations couvert par cet article demeure toutefois relativement étroit puisqu'il ne concerne qu'un petit nombre de stagiaires de sexe masculin du secondaire et que les modifications mesurées ici n'ont touché que la fréquence des comportements de prévention, d'organisation et de réaction. D'autres analyses plus approfondies partant du même corpus de données devraient permettre de mettre en tension d'autres variables comme l'intensité, la durée et la nature des comportements d'indiscipline (Polirstok, 2015) ainsi que le sexe des élèves puisqu'il ressort, suivant Bonniot-Paquiem, Cogérino \& Champely (2009), que les garçons sont, plus que les filles, la cible d'interventions rapides même s'il est question de comportements qualifiés de passifs. Ces nouvelles analyses, si elles intégraient la variable du temps d'apprentissage cognitif et moteur (Brunelle, Tousignant \& Godbout, 1996) pourraient également jeter un éclairage plus précis sur l'évolution du climat d'apprentissage qui a régné dans les classes dirigées par les stagiaires des deux groupes entre le début et la fin du stage terminal de la formation initiale à l'enseignement de l'EP. Enfin, nous pensons que les résultats présentés dans cet article devraient inviter les responsables de la formation initiale des enseignants d'EP de Tunisie à réviser les programmes de manière à y inclure systématiquement des activités pédagogiques signifiantes portant sur la gestion de classe et la gestion de l'indiscipline, mais aussi à fournir, comme le proposent Lavay et al. (2012), des expériences de stage diversifiées, supervisées et bien arrimées au curriculum de formation. Sur ce dernier point, nous savons pertinemment qu'en Tunisie tout espoir de réforme doit s'inscrire dans une perspective réaliste pour au moins deux raisons essentiellement liées à la résistance au changement des universitaires chargés de la formation des enseignants. D'abord, nous faisons l'hypothèse que, comme dans l'étude de Johnson (2005), les formateurs universitaires tunisiens seront, malgré les évidences rapportées plus haut, peu nombreux à soutenir l'idée que la gestion de classe constitue l'un des préalables les plus importants à la réussite scolaire des élèves. Ensuite, nous basant sur des travaux antérieurs (Desbiens, Fraj \& Bouzid, 2016) et actuels (Fraj, 2019) à propos de la dernière réforme des programmes tunisiens de formation à l'enseignement de l'EP, nous redoutons que tout nouvel effort de réforme des programmes se heurte à la résistance des formateurs universitaires pour n'aboutir dans les faits qu'à un statut quo où l'intervention éducative et à la pédagogie avec12,68 \% du volume horaire global ou 25 crédits continueront à faire figure d'enfant pauvre. 


\section{RÉFÉRENCES}

Amamou, S., Desbiens, J. F., Spallanzani, C. \& Vandercleyen, F. (2017). La perception du sentiment d'efficacité personnelle à gérer la classe par des enseignants stagiaires au milieu de leur formation en éducation physique. Revue PhénEps, 9(1), 1-18.

Archambault, J. \& Chouinard, R. (2016). Vers une gestion éducative de la classe (4e éd), Boucherville : Gaëtan Morin Éditeur

Archambault, J. et Chouinard, R. (2009). Vers une gestion éducative de la classe (3e éd.). Boucherville, Canada : Gaëtan Morin Éditeur.

Atici, M. (2007). A small-scale study on student teachers' perceptions of classroom management and methods for dealing with misbehavior. Emotional and behavioral difficulties, 12(1), 15-27.

Bali, N. (2015). The Tunisians Cooperative Teachers and Student Teachers' Conceptions about Class Management Skill. Creative Education, 6, 87-99.

Bandura, A. (2007). Auto-efficacité : le sentiment d'efficacité personnelle (2ème éd), (J. De Lecomte, Trans). Paris: De Boeck.

Bonniot, Paquiem, N., Cogérino, G. \& Champely, S. (2009). Les enseignants d’ÉPS face aux élèves qui décrochent de l'activité : interventions selon le sexe des élèves et discours relatifs aux comportements observés. Revue STAPS, 84, 77-92. Doi : 10.3017/sta.084.0077.

Brunelle, J., Tousignant, M. \& Godbout, P. (avec la collaboration de Spallanzani, C., Brunelle, J. P., Martel, D., Trudel, P., Gagnon, J., Savard, C. \& Rami, A.) (1996). Le temps d'apprentissage. Sainte-Foy : GRIAP, département d'éducation physique, Université Laval.

Chouinard, R. (1999). Enseignants débutants et pratique de gestion de classe. Revue des sciences de l'éducation $X X V(3)$, p. $497-514$.

Desbiens, J. F. (2002). L'évaluation et la responsabilisation des élèves : ébauche d'une problématique sur le thème de la supervision active en enseignement de l'éducation physique. Brock Education, 12(1), 36-48.

Desbiens, J. F., Bali, N., Spallanzani, C., Vandercleyen, F., \& Beaudoin, S. (2018). Validation d'un instrument pour mesurer les préoccupations d'enseignants stagiaires en ÉPS tunisiens. Anadolu University Journal of Education Faculty (AUJEF), 2(2), 158-177.

Desbiens, J. F., Fraj, H. et Bouzid, M. S. (2016). Défis et nécessité d'une formation professionnalisante des intervenants tunisiens en éducation physique et en sports. Conférence principale présentée au $2^{\text {ème }}$ congrès international des sciences du sport de l'Observatoire national du sport de Tunisie, 29 avril, Hammamet, Tunisie.

Desbiens, J. F., Lanoue, S, Turcotte, S, Tourigny, J. S., \& Spallanzani, C. (2009). Perception de la fréquence d'apparition des comportements perturbateurs par des stagiaires en enseignement de l'éducation physique et à la santé (EPS). Nouveau cahier de la recherche en éducation, 12(2), p .179-193.

Desbiens, J. F., Turcotte, S., Spallanzani, C., Roy, M., Brunelle, J. P., Tourigny, J. S., \& Lanoue, S. (2008). Une analyse des comportements perturbateurs survenus durant des cours d'éducation physique et à la santé enseignés par des stagiaires, De Boeck Supérieur Staps, Vol.3 - n 81, 73-88.

Desbiens, J. F., Turcotte, S., Spallanzani, C., Roy, M., Tourigny, J. S., \& Lanoue, S. (2011). Comment des stagiaires en enseignement de l'éducation physique et à la santé (EPSa) réagissent-ils face à l'indiscipline de leurs élèves ? Science \& Motricité 73, 39-54, Canada.

Doyle, W. (1986). Classroom organization and management. In M. C. Wittrock (Ed.), Handbook of Research on Teaching: (3rd ed., pp. 392-431). New York: Macmillan.

Evertson C. M., \& Weinstein, C. (2006). Classroom management as a field of inquiry. In C. M. Evertson \& C. Weinstein (Eds.), Handbook of Classroom Management: Research, practice, and contemporary issues (pp. 3-15). Mahwah, NJ: Lawrence Erlbaum Associates, Inc.

Fortin, M. F. (2010). Fondements et étapes du processus de recherche : Méthodes quantitatives et qualitatives (2ème éd.). Chenelière Education. 
Fraj, H. (2019). La formation initiale à l'enseignement de l'ÉPS en Tunisie après l'implantation du modèle LMD : point de vue des acteurs sur l'ancien et le nouveau curriculum ainsi que sur la professionnalisation (Thèse de doctorat inédite). Université de Sherbrooke, Sherbrooke, Canada.

Freeman, S., Eddy, S. L., McDonough, M., Smith, M. K., Okoroafor, N., Jordt, H., \& Wenderoth, M. P. (2014). Active learning increases student performance in science, engineering, and mathematics, $P N A S$, $111(23) 8410-8415$.

Gaudreau, N. (2017). Gérer efficacement sa classe. Les cinq éléments essentiels. Québec: Presses de l'Université du Québec.

Gauthier, C., Desbiens, J. F., Malo, A., Martineau, S. et Simard, D. (1997). Pour une théorie de la pédagogie. Recherches contemporaines sur le savoir des enseignants. Saint-Nicolas : Les Presses de l'Université Laval.

Gouvernement du Québec. (2015). Cadre de référence et guide à l'intention du milieu scolaire. L’intervention auprès des élèves ayant des difficultés de comportement. Québec: Ministère de l'Éducation, de l'Enseignement supérieur et de la Recherche. http://www.education.gouv.qc.ca/fileadmin/site web/documents/education/adaptation-scolaire-services comp/14_00479 cadre intervention_élèves_difficultes_comportement.pdf

Goyette, R., Doré, R., \& Dion, É. (2000). Pupils' misbehavior and the reactions and causal attributions of physical education student teachers: a sequential analysis. Journal of Teaching in Physical Education, 20 (3), 3-14.

Hattie, J. (2012). Visible learning for the teachers. Maximizing impact on learning. Londres : Routhledge.

Hodges, Kulinna, P. (2008). Models for Curriculum and Pedagogy in Elementary School Physical Education. The Elementary School Journal, 108(3), 219-227.

Howel, D. C. (2008). Méthodes statistiques en sciences humaines. (2ème éd), De Boeck Université.

Johnson, J. (2005). Isn't it time for school of education to take concerns about student discipline more seriously? Teachers College Record. Document récupéré le 14 février 2006 du site Web du Teacher College Record: http://www.tcrecord.org.

Kearney, P. (1987). Experienced and prospective teachers' compliance-gaining message selections on common student misbehaviors. $73^{\mathrm{e}}$ Congress of Speech Communication Association, Boston, 5-8 November, 184290.

Kounin, J. S. (1970). Discipline and group management in classroom. New York: Holt, Rinehart \& Winston.

Lavay, B., Henderson, H., French, R. \& Guthrie, S. (2012). Behavior management instructional practices and content of college/university physical education teacher education (PETE) program. Physical education and sport pedagogy, 17(2), 195-210. doi: doi.org/10.1080/17408989.2010548063.

Leriche, J., Desbiens, J. F., Amade-Escot, C. et Tinning, R. (2016). Compatibility and complementarity of classroom ecology paradigm and didactique research traditions in physical education. QUEST. DOI: 10.1080/00336297.2016.1144516.

Levin, J., \& Nolan, J. F. (2000). Principles of Classroom Management: A Professional Decision-Making Model. Boston, MA: Allyn and Bacon.

Maddeh, T., Hermessi, S., Bennour, N., \& Souissi, N. (2015a). Student Teachers' Reactions Responding to Students' Disruptive Behaviors: A Case Study in High School Education in Tunisia. Creative Education, 6 (11). https://DOI: $10.4236 /$ ce.2015.611110

Maddeh, T, Desbiens, J. F, \& Souissi, N (2018) Effets d'une formation complémentaire sur la compétence d'enseignants stagiaires d'éducation physique et sportive tunisiens à prévenir et à gérer l'indiscipline. Mov Sport Sci/Sci Mot, https://doi.org/10.1051/sm/2018018.

Maddeh, T., Bennour, N, \& Souissi, N. (2015b). Study of students' disruptive behavior in high-school education in physical education classes. Advances in Physical Education (APE), 5(3). https://DOI: 10.4236/ape.2015.53018

Nault, T. et Lacourse, F. (2016). La gestion de classe: une compétence à développer ( $2^{e}$ éd.). Anjou: Les Éditions CEC. 
Nault,T., \& Fijalkow, J. (1999). Introduction. La gestion de la classe : d'hier à demain. Érudit, Revue des sciences de l'éducation, 25(3), p. 451-466.

Opinel, M. (2001). Incidence de la tâche des novices du secondaire sur la compétence à gérer une classe. Mémoire de maîtrise à la Faculté d'éducation non publié. Sherbrooke,

Polirstok, S. (2015). Classroom management strategies for inclusive classrooms. Creative Education, 6, $927-$ 933.

Postholm, M. B. (2013). Classroom management: what does research tell us ? European Educational Research Journal, 12(3), 389-402.

Sieber, M. (2001). Comment gérer l'indiscipline en classe: Gérer l'indiscipline auprès d'élèves hyperactif oppositionnels et provocateurs. Edition universitaire Fribourg, Souisse. Vol. 11

Siedentop, D. (1994). Apprendre à enseigner l'éducation physique. Traduction et adaptation de M. Tousignant, P. Boudreau et A. Fortier. Montréal: Gaëtan Morin éditeur.

Tardif, M. \& Lessard, C. (1999). Le travail enseignant au quotidien. Sainte-Foy : Presses de l'Université Laval. 


\section{EXTENDED ABSTRACT}

The management of undisciplined behaviors (UB) in the classroom represents a considerable source of worry as well as a challenge for all teachers (Desbiens et al., 2001: Habak et al., 2016). According to Gaudreau (2017), the management of pupils' UB may take almost $50 \%$ of teaching time. When teacher trainees (TT) do not find a viable solution to indiscipline, this may lead to the settling in amongst them of a feeling of lack of motivation about teaching (Desbiens et al., 2018). This may also lead to the progressive loss of self-confidence in their autonomy and proficiency (Amanou et al., 2017: Bandura, 2007).

Desbiens et al. (2018) and Desbiens et al. (2008) report that teacher trainees are not well-prepared in their training to prevent and react efficiently to UB. Accordingly, teacher training institutions are encouraged to improve and revise their training curriculum in order to get teachers prepared to cope in a strategic way with pupils' UB (Maddeh et al., 2018, Freeman et al., 2014; Desbiens et al., 2011). According to Gaudreau (2019), the time allotted to the acquisition of difficult behavior management guidelines in initial teacher training in 11 countries, hardly corresponds to $3,4 \%$ of the average time allotted to the training as a whole (Gaudeau, 2019). In Tunisia, the initial courses for students majoring in physical education haven't got any part that is specifically devoted to the prevention as well as management of UB (Maddeh et al., 2015; Maddeh et al., 2015). Consequently, it seemed highly appropriate to put forward a supplementary training in the prevention and management of UB based on Sieber's 2001 theoritical framework. The latter puts forward three main ways to indiscipline management: 1) preventive management, 2) organizational management, and 3) reactive management. This paper looks into the effects of a supplementary training of Tunisian teacher trainees in sports and physical education on their ability to prevent and deal with UB. Its general objective is to describe and analyze the supplementary training's effects on the way UB is dealt with by trainees. More specifically, it consists in the comparison of the experimental (exp. GR) and the pilot groups' (plt. GR) frequency of the episodes of preventive, reactive, and organizational management. And this will be done in three sampling moments during the school year (at the beginning (T1), during (T2), and at the end of the training (T3)).

The supervision to which the exp. GR trainees have been subject lasts for the whole graduating year of the initial PE teacher training program at ISSEP Ksar Said, Tunisia. The training is composed of two parts: a theoretical one and a practical one. The supervision program's main theme is the prevention and management of UB in PE classes. The sample is composed of ten undergraduate trainees registered in third year (graduating year) bachelor in Physical education at ISSEP Ksar Said, Tunisia. The group is subdivided into two groups of five each. Five trainees form the exp.GR and five others form the control.GR. Audio-visual records of lessons piloted by trainees $(n=10)$ have been made in the three different moments. A video record has been made in order to encode the episodes of preventive, reactive, and organizational management of indiscipline. Inter-encoding reliability has been checked. A high degree of reliability has been obtained. In order to better appreciate the UB frequency degree in classes managed by trainees, intra and inter-group comparisons have been made in relation to the above mentioned three moments of sampling using parameter-free statistics.

The results show that instances of the use of prevention and organization behavior by trainees of both groups go higher at the end of the training compared to the beginning. However, this tendency is significantly higher among the exp.GR than the control.GR. On the other hand, we notice a sinificant decrease in reactive behavior in the two groups. This is far more noticeable among the TT of the exp.GR than among the TT of the control.GR. Another outcome is that the effects of the supplementary training on the frequency of the manifestation of UB are not uniform. A significant difference in favor of the exp.GR trainees is only reported at the end of the training concerning the organizational management behaviors. On the other hand, the effects of the supplementary training on the frequency of the TT behaviors were not uniformly manifested. A significant difference in favor of the Exp.GR was only noticed at the end of the practicum for organizational management while two differences in favor of the same group for prevention management were reported in the middle and at the end of the practicum.

This study shows that a supplementary training complementing the initial one in prevention and UB management could allow Tunisian PE trainees to significantly alter the way they intervene in a positive manner in response to pupils' UB. The results of this study should be considered as an invitation to the officials responsible for the initial PE teacher training in Tunisia as well as in other countries to revise their curricula in such a way as to include systematically meaningful pedagogical activities on classroom management and indiscipline's management and provide also, as Lavay, Henderson and French Guthrie (2012) suggest, varied and supervised trainings that is adjusted to the training curriculum. education.

Keywords: complimentary training, trainee teachers, prevention and management of indiscipline, physical 


\section{Annexe 1}

Présentation des modes de la gestion des comportements perturbateurs de Sieber (2001, p. 105-120)

\begin{tabular}{|c|c|c|c|}
\hline Les modes de gestion & \multicolumn{2}{|c|}{ Formes de gestion } & Les actes qui leur sont associés \\
\hline \multirow{4}{*}{$\begin{array}{c}\text { La gestion } \\
\text { préventive } \\
\text { Regroupe l'ensemble } \\
\text { des mesures et } \\
\text { techniques } \\
\text { d'intervention qui } \\
\text { visent à prévenir } \\
\text { l'apparition de } \\
\text { comportements } \\
\text { indisciplinés lors des } \\
\text { cours d'EP }\end{array}$} & \multirow[t]{3}{*}{$\begin{array}{l}\text { Les intermèdes } \\
\text { préventifs }\end{array}$} & $\begin{array}{l}\text { La gestion collective } \\
\text { avec l'ensemble du } \\
\text { groupe-classe }\end{array}$ & $\begin{array}{l}\text { L'enseignant élabore et met en œuvre selon } \\
\text { différentes stratégies plus ou moins participatives } \\
\text { des règles de vie auprès de l'ensemble des élèves. } \\
\text { La présentation des règles a généralement lieu en } \\
\text { début d'année scolaire, au début d'un stage, mais } \\
\text { peuvent être reprises ponctuellement notamment } \\
\text { lors du retour de longs congés. Ces échanges entre } \\
\text { enseignant et élèves qui peuvent être plus libres ou } \\
\text { s'inscrire dans des dispositifs plus formalisés } \\
\text { comme le conseil de coopération. }\end{array}$ \\
\hline & & $\begin{array}{l}\text { La gestion } \\
\text { individualisée avec } \\
\text { l'élève jugé comme } \\
\text { indiscipliné }\end{array}$ & $\begin{array}{l}\text { L'enseignant prend à part un élève et s'entretient } \\
\text { avec lui de son comportement et de l'aide qu'il } \\
\text { peut lui fournir. À titre d'exemple, il peut convenir } \\
\text { avec lui de signes non verbaux (ex. : variation du } \\
\text { rapport de proximité, signe de la main, toucher } \\
\text { adéquatement l'élève, varier l'intonation, etc.) } \\
\text { qu'il lui adressera en situation d'enseignement et } \\
\text { qui lui permettront de modifier son comportement. }\end{array}$ \\
\hline & & $\begin{array}{l}\text { La gestion en } \\
\text { collaboration avec les } \\
\text { parents de l'élève }\end{array}$ & $\begin{array}{l}\text { L'enseignant discute hors du temps de classe avec } \\
\text { les parents du comportement de leur enfant afin } \\
\text { d'obtenir leur soutien dans une perspective de } \\
\text { modification du ou des comportements } \\
\text { problématiques de l'élève. }\end{array}$ \\
\hline & $\begin{array}{l}\text { La gestion } \\
\text { préventive intégrée à } \\
\text { l'enseignement } \\
\text { (p.107) }\end{array}$ & $\begin{array}{l}\text { Fait partie de } \\
\text { l'enseignement des } \\
\text { matières pédagogiques } \\
\text { dans la mesure où elle a } \\
\text { lieu avant une activité } \\
\text { pédagogique (p. 109) }\end{array}$ & $\begin{array}{l}\text { C'est la prévention en cours d'enseignement. Elle } \\
\text { s'adresse à tout le groupe-classe. L'enseignant } \\
\text { rappelle aux élèves ses attentes avant de } \\
\text { commencer une leçon ou d'entamer une activité. }\end{array}$ \\
\hline $\begin{array}{l}\text { Réfère à la manière } \\
\text { dont l'enseignant } \\
\text { organise sa classe et } \\
\text { son enseignement ( } \mathrm{p} . \\
\text { 105) }\end{array}$ & Spatiale & $\begin{array}{c}\text { Touche à } \\
\text { l'aménagement de la } \\
\text { salle de classe. Il tient } \\
\text { compte du nombre } \\
\text { d'élèves, des types de } \\
\text { comportements } \\
\text { indisciplinés et de } \\
\text { l'intégration sociale de } \\
\text { l'élève (p. 109) }\end{array}$ & $\begin{array}{l}\text { Réfère à / au(x): } \\
\text { - la planification et la mise en œuvre de } \\
\text { l'aménagement du plateau de travail pour } \\
\text { s'assurer de la disponibilité du matériel, de } \\
\text { l'adéquation de sa disposition et de la sécurité } \\
\text { - la planification et mise en œuvre de } \\
\text { l'organisation sociale, de l'emplacement des } \\
\text { élèves sur le plateau selon les différentes } \\
\text { étapes de la leçon et des configurations de }\end{array}$ \\
\hline
\end{tabular}




\begin{tabular}{|c|c|c|c|}
\hline & & & $\begin{array}{l}\text { travail } \\
\text { - les procédures et les consignes planifiées et } \\
\text { mise en œuvre pour gérer les épisodes de } \\
\text { transition et de battement avant, pendant et } \\
\text { après le cours } \\
\text { - au positionnement stratégique ainsi qu'aux } \\
\text { déplacements de l'enseignant sur le plateau de } \\
\text { travail }\end{array}$ \\
\hline & Temporelle & $\begin{array}{l}\text { Consiste à susciter la } \\
\text { motivation, l'attention } \\
\text { et l'engagement des } \\
\text { élèves vis-à-vis des } \\
\text { activités pédagogiques. } \\
\text { Elle découle } \\
\text { directement de l'analyse } \\
\text { systémique de la classe. } \\
\text { (p. } 110 \text { ) }\end{array}$ & $\begin{array}{l}\text { - } \quad \text { Minimiser les temps morts } \\
\text { - } \quad \text { Déterminer l'ordre de succession des } \\
\text { - } \quad \text { Orgments de leçons et varier leur forme } \\
\text { - } \quad \text { Enseigner l'organisation } \\
\text { - } \quad \text { Partager d'attention }\end{array}$ \\
\hline \multirow{4}{*}{$\begin{array}{l}\text { La gestion réactive } \\
\text { Regroupe l'ensemble } \\
\text { des mesures et des } \\
\text { techniques } \\
\text { d'intervention qui } \\
\text { sont mobilisés en } \\
\text { réaction au } \\
\text { comportement } \\
\text { dérangeant d'un } \\
\text { élève ou de plusieurs } \\
\text { élèves en vue de le } \\
\text { faire cesser ou de le } \\
\text { modifier }\end{array}$} & \multicolumn{2}{|c|}{$\begin{array}{l}\text { 1. Gestion par remontrances } \\
\text { Concerne les interventions dirigées vers le } \\
\text { comportement d'un élève et suppose une } \\
\text { fracture de la continuité pédagogique. }\end{array}$} & $\begin{array}{l}\text { Réfère à des prescriptions, des remontrances, des } \\
\text { réprobations, des menaces, des punitions centrées } \\
\text { sur le comportement et adressées publiquement à } \\
\text { l'élève (p. 118) }\end{array}$ \\
\hline & \multicolumn{2}{|c|}{$\begin{array}{l}\text { Concerne les interventions dirigées vers le } \\
\text { comportement d'un élève et suppose une } \\
\text { fracture de la continuité pédagogique. }\end{array}$} & $\begin{array}{l}\text { Réfère à des explications, des demandes de } \\
\text { clarification centrées sur le comportement d'un ou } \\
\text { de plusieurs élèves et adressées publiquement à la } \\
\text { ou aux personnes concernées (p. 118). }\end{array}$ \\
\hline & \multicolumn{2}{|c|}{$\begin{array}{l}\text { Concerne les interventions dirigées vers un } \\
\text { élève. Leur contenu porte sur la tâche scolaire. } \\
\text { Elles supposent la préservation de la continuité } \\
\text { pédagogique. }\end{array}$} & $\begin{array}{l}\text { Prescriptions, demandes, messages d'évaluation } \\
\text { centrés sur les apprentissages et adressés } \\
\text { publiquement à l'élève (p. 118) }\end{array}$ \\
\hline & \multicolumn{2}{|c|}{$\begin{array}{l}\text { 4. Gestion indirecte } \\
\text { Concerne les interventions dirigées vers un } \\
\text { groupe ou sous-groupe d'élèves. Leur contenu } \\
\text { porte sur le comportement. Elles supposent une } \\
\text { fracture de la continuité pédagogique. }\end{array}$} & $\begin{array}{l}\text { Prescriptions, remontrances, réprobations, } \\
\text { menaces, punitions, explications, demandes de } \\
\text { clarification centrées sur le comportement et } \\
\text { adressées à un groupe d'élèves (P. 118) }\end{array}$ \\
\hline
\end{tabular}

